\title{
Aspectos Fonoaudiológicos em Pacientes Diagnosticados com Meningioma antes e após 0 Tratamento Cirúrgico
}

\author{
doi: https://doi.org/10.32635/2176-9745.RBC.2018v64n2.87 \\ Oral Miofunctional Aspects of Patients Diagnosed with Meningioma before and after Surgical Treatment \\ Aspectos Fonoaudiológicos en Pacientes Diagnosticados con Meningioma antes y después del Tratamiento \\ Quirúrgico
}

Caroline Sousa Costa'; Renata D'Arc Scarpel'; Ana Terra Brito de Jesus ${ }^{3}$

Resumo

Introdução: Os meningiomas estão entre as neoplasias intracranianas primárias mais frequentes e, entre as deficiências neurológicas causadas, estão as alteraçôes fonoaudiológicas, principalmente os déficits de deglutição e comunicação. Com isso, estudos que identifiquem precocemente as alteraçôes fonoaudiológicas são necessários para uma adequada intervençâo terapêutica. Este estudo tem como objetivo descrever os aspectos fonoaudiológicos em pacientes diagnosticados com meningioma antes e após o tratamento cirúrgico. Relato dos casos: Participaram da pesquisa seis indivíduos diagnosticados com meningioma internados em um hospital geral de Salvador - BA. Os participantes foram submetidos à avaliação fonoaudiológica antes e após a ressecção do tumor, com aplicação do Protocolo de avaliação clínica fonoaudiológica à beira leito. No momento pré-cirúrgico, as alteraçóes fonoaudiológicas ocorreram em um número pequeno de casos, estando relacionadas principalmente a déficits leves de motricidade orofacial e disfagia de leve a moderada. Já no período pós-operatório, as alteraçôes fonoaudiológicas foram frequentes, principalmente quanto à deglutição, com involução da Escala funcional de ingestáo por via oral e necessidade de terapia fonoaudiológica em todos os casos. Conclusáo: Conclui-se que o meningioma pode acarretar diferentes alteraçôes fonoaudiológicas, principalmente quanto à deglutiçâo, sendo mais frequente no momento pós-cirúrgico em razão da manipulação dos vasos e nervos, e mais preocupante em regióes cranianas posteriores.

Palavras-chave: Meningioma; Transtornos de Deglutição; Linguagem; Neoplasias Encefálicas.

\begin{abstract}
Introduction: Meningiomas are among the most frequent primary intracranial neoplasms, and among the neurological deficiencies caused are speech-language disorders, mainly deglutition and communication deficits. Thus, studies that identify early speech-language disorders are necessary for an adequate therapeutic intervention. This study aims to describe speech-language pathology in patients diagnosed with meningioma before and after surgical treatment. Case report: Six individuals with meningioma diagnostics participated in the study hospitalized in a general hospital in Salvador - BA. Participants were submitted to speech and language assessment before and after tumor resection, with application of the protocol of clinical evaluation of speech-language pathology. At the pre-surgical stage, speech-language disorders occurred in a small number of cases, mainly related to mild deficiencies of orofacial motor and mild to moderate dysphagia. Already in the postoperative period, speech-language disorders were frequent, especially regarding swallowing, with involution of the functional oral intake scale and need for speech therapy in all cases. Conclusion: It is concluded that the meningioma can cause different speech-language disorders, mainly in swallowing, being more frequent in the post-surgical moment due to the manipulation of the vessels and nerves, being more worrying in posterior cranial regions.

Key words: Meningioma; Deglutition Disorders; Language; Brain Neoplasms.
\end{abstract}

Resumen

Introducción: Los meningiomas están entre las neoplasias intracraneales primarias más frecuentes y entre las deficiencias neurológicas causadas están las alteraciones fonoaudiológicas, principalmente los déficit de deglución y comunicación. Con ello, estudios que identifiquen precozmente las alteraciones fonoaudiológicas son necesarios para una adecuada intervención terapéutica. Este estudio tiene como objetivo describir los aspectos fonoaudiológicos en pacientes diagnosticados con meningioma antes y después del tratamiento quirúrgico. Relato de casos: Participaron de la investigación seis individuos diagnosticados con meningioma internados en un hospital general de Salvador - BA. Los participantes fueron sometidos a la evaluación fonoaudiológica antes y después de la resección del tumor, con aplicación del protocolo de evaluación de evaluación clínica fonoaudiológica al borde lecho. En el momento prequirúrgico las alteraciones fonoaudiológicas ocurrieron en un número pequeńo de casos, estando relacionadas principalmente a los déficit leves de motricidad orofacial y disfagia de leve a moderada. En el período postoperatorio. las alteraciones fonoaudiológicas fueron frecuentes, principalmente en cuanto a la deglución, con involución de la escala funcional de la ingestión por vía oral y necesidad de terapia fonoaudiológica en todos los casos. Conclusión: Se concluye que el meningioma puede acarrear diferentes alteraciones fonoaudiológicas, principalmente en cuanto a la deglución, siendo más frecuente en el momento post-quirúrgico debido a la manipulación de los vasos y nervios, siendo más preocupantes en regiones craneales posteriores. Palabras clave: Meningioma; Transtornos de Deglución; Lenguaje; Neoplasias Encefálicas.

\footnotetext{
${ }^{1}$ Fonoaudióloga. Especialista em Oncologia pelo Departamento Ciências da Vida da Universidade do Estado da Bahia. Salvador (BA), Brasil. E-mail: carolcostafga@gmail.com. Orcid ID: https://orcid.org/0000-0002-2117-6537

${ }^{2}$ Fonoaudióloga. Doutora em Processos Interativos dos Órgãos e Sistemas. Docente Titular do Departamento Ciências da Vida da Universidade do Estado da Bahia. Salvador (BA), Brasil. E-mail: renatascarpel@gmail.com. Orcid ID: https://orcid.org/0000-0002-5068-9008

${ }^{3}$ Fonoaudióloga. Especialista em Nutrição Clínica e em Saúde Pública. Supervisora do Serviço de Fonoaudiologia do Hospital Geral Roberto Santos. Salvador (BA), Brasil. E- mail: fga.anaterra@gmail.com. Orcid ID: https://orcid.org/0000-0002-7961-283x

Endereço para correspondência: Caroline Sousa Costa. Rua José Olímpio da Silva, 5 - IAPI. Salvador (BA), Brasil.
} 


\section{INTRODUÇÃO}

Os tumores do sistema nervoso central são um conjunto de neoplasias originárias de células de sustentação do tecido nervoso. Incluem os tumores do encéfalo, das meninges cranianas e dos nervos intracranianos ${ }^{1}$. São tumores raros, correspondendo a $2 \%$ dos todos os cânceres conhecidos, porém com elevada mortalidade em adultos ${ }^{2}$.

Entre as neoplasias intracranianas primárias, os meningiomas estão entre os mais frequentes ${ }^{2}$, representando aproximadamente $15 \%$ de todas as neoplasias intracranianas ${ }^{3}$. São tumores extra-axiais conhecidos por ocorrer em vários locais dentro do cérebro, originados a partir das células meningoteliais aracnoideas ${ }^{4-5}$.

Geralmente, são benignos (grau I), bem circunscritos, lentos e curáveis por cirurgia, dependendo da localização; contudo, podem ter comportamento mais agressivo, sendo classificados como grau II (atípico), cerca de 7\% dos casos, ou grau III (anaplásico ou maligno), 3\% dos $\operatorname{casos}^{6}$. Ocorrem com mais frequência em mulheres, adultos e idosos, sendo rara sua apresentação na infância ${ }^{5}$.

Os tumores cerebrais originam inúmeros comprometimentos neurológicos que variam em relaçáo ao seu tamanho, sítio da lesão e a característica invasiva do tumor $^{4,7}$. Alguns estudos internacionais apontam a afasia e a disfagia como complicaçóes em casos de neoplasias meníngeas ${ }^{8-9}$.

A aspiração traqueal decorrente das dificuldades de deglutição é um sintoma importante que deve ser considerado, podendo acarretar prejuízos ao estado pulmonar, aspectos nutricionais, desidratação, perda de peso, pneumonia e morte ${ }^{10}$. As alteraçôes na comunicação também podem acarretar impactos negativos, pois, além de afetar a linguagem, interferem nos processos relacionados.

A reabilitação precoce é importante para prevenir complicaçôes e maximizar a capacidade funcional do paciente, melhorando sua qualidade de vida ${ }^{4}$. Com isso, estudos que identifiquem precocemente as alteraçóes fonoaudiológicas são necessários para uma intervenção terapêutica especializada. Portanto, o objetivo desta pesquisa é descrever os aspectos fonoaudiológicos em pacientes diagnosticados com meningioma antes e após o tratamento cirúrgico.

\section{RELATO DOS CASOS}

Trata-se de um estudo longitudinal descritivo em série de casos, desenvolvido na enfermaria de Neurocirurgia e Unidade de Terapia Intensiva (UTI) Neurológica do Hospital Geral Roberto Santos (HGRS). O estudo foi constituído por casos de indivíduos diagnosticados com meningioma e com proposta de tratamento cirúrgico. Foram excluídos aqueles que possuíam outra doença neurológica, alteração fonoaudiológica prévia ao diagnóstico de meningioma, os que passaram por intubação orotraqueal prolongada (maior que $24 \mathrm{~h}$ ) e aqueles que tiveram dados incompletos.

Foi realizada uma busca ativa para recrutar esses casos no período de novembro de 2017 a janeiro de 2018 e, após análise de prontuário médico e dos critérios de participação para o estudo, foi realizada avaliação fonoaudiológica, quando em quadro clínico estável, antes e após a cirurgia de ressecção tumoral.

A avaliação fonoaudiológica foi realizada a partir do "Protocolo de avaliaçáo clínica fonoaudiológica à beira leito" (Anexo 1), estabelecido pelo Serviço de Fonoaudiologia do HGRS (baseado no protocolo fonoaudiológico de avaliaçáo do risco para disfagia $(\mathrm{PARD}){ }^{10}$. Em avaliação fonoaudiológica pré e pós-cirúrgica, foram pesquisados os seguintes aspectos: nível de consciência; respiração; via de alimentação, triagem de comunicação (compreensão e expressão) a partir do discurso espontâneo, fala automática, nomeação e repetição; avaliação de órgãos fonoarticulatórios; qualidade vocal por meio da Escala GRBASI ${ }^{11}$; avaliaçáo subjetiva da tosse como parâmetro de proteção de vias aéreas; avaliação da deglutiçẫo de saliva; avaliação direta da deglutição (com dieta); classificação da Escala funcional de ingestão por via oral $(\mathrm{FOIS})^{12}$; e, ao fim da avaliação, foi definida conduta fonoaudiológica quanto à via de alimentaçáo. Além disso, foram analisados fatores sociodemográficos, localização do tumor e complicações cirúrgicas, baseados nas informaçôes do prontuário individual do paciente. Os dados coletados foram tabulados e as variáveis foram analisadas quantitativamente. Em função do pequeno número de casos, não se realizou análise estatística.

Este estudo foi aprovado pelo Comitê de Ética em Pesquisa do HGRS, sob o número de parecer 2.381.369 e incluiu o Termo de Consentimento Livre e Esclarecido (TCLE) por escrito, de acordo com os padrōes éticos da Resolução no. 466/2012 do Conselho Nacional de Saúde.

Foram recrutados para a pesquisa seis casos de meningioma, com predominância do sexo feminino, sendo quatro mulheres e dois homens, com idade variando de 37 a 74 anos (média de idade de 55 anos).

Os dados sociodemográficos referentes à escolaridade mostram que cinco casos eram compostos por indivíduos com ensino fundamental incompleto e apenas um caso com segundo grau completo. Quanto à profissão, foram recrutados dois lavradores, duas donas de casa, uma microempresária e um borracheiro.

Dos seis casos, três tiveram o meningioma localizado em regiáo cortical frontal, um caso em asa do esfenoide esquerda 
(com compressão das regiôes frontal e temporal), um caso em tubérculo selar e um em fossa craniana posterior.

Com relação ao procedimento cirúrgico, em três casos não foram observadas complicaçôes cirúrgicas, enquanto dois apresentaram sangramento moderado durante a craniotomia e, em um caso, não foi possível realizar a ressecção total do tumor por envolvimento de vasos.

No momento pré-cirúrgico, todos os casos encontravam-se em adequado nível de consciência, em ventilação espontânea ao ar ambiente. Quanto à via de alimentação, cinco casos encontravam-se em dieta normal por via oral exclusiva e, em um caso, fazia-se uso de sonda nasoenteral como via alternativa de alimentação exclusiva. Com relação à comunicaçáo, em todos casos foi observado compreensão sem alteraçôes e, quanto à expressão, apenas em um caso foram observados déficits.

$\mathrm{Na}$ avaliação de órgãos fonoarticulatórios, quatro casos cursaram com musculatura facial sem alteraçôes, um caso apresentou paresia facial à direita e um apresentou paresia facial à esquerda. À oroscopia, foram observados edentulismo parcial, edentulismo total e uso de prótese dentária em dois casos em cada evento supracitado, sem evidência de outras alteraçóes. Quanto à força e mobilidade de estruturas orofaciais (lábios, língua e bochechas), quatro casos apresentaram aspectos dentro dos padrôes de normalidade, um apresentou todos os parâmetros alterados e um apresentou redução de mobilidade de bochechas e lábios.

Em avaliação de deglutição de saliva, todos os casos apresentaram frequência de deglutição de saliva espontânea adequada, com ausculta cervical limpa. A tosse sob comando foi considerada fraca em apenas um caso, a qualidade vocal foi classificada grau global 0 para cinco casos e grau global 1 com discreta rugosidade em um caso, de acordo com a Escala GRBASI.

Foi possível realizar avaliação direta da deglutição em todos os casos. Os resultados encontrados foram descritos na Tabela 1. Três casos obtiveram diagnóstico de deglutição normal, um foi diagnosticado com deglutição funcional, um com disfagia leve e um com disfagia moderada.

Foi aplicada a FOIS, com base na conduta após avaliação fonoaudiológica. Quatro casos foram classificados como nível 7 (via oral total sem restriçôes), um caso foi classificado como nível 6 (via oral total com múltiplas consistências, porém com restriçôes alimentares) e um caso foi classificado como nível 2 (dependente de via alternativa e mínima via oral de alimento ou líquido). Foi liberada dieta oral em todas as consistências sem restriçôes para quatro casos. Para um, foi liberada dieta oral até a consistência semissólida e, para outro, foi mantida alimentação via sonda nasoenteral e contraindicada dieta oral (treino de via oral em fonoterapia).
Tabela 1. Alterações da deglutição em avaliação direta pré-cirúrgica

\begin{tabular}{l|l|l} 
Caso & \multicolumn{1}{|c|}{$\begin{array}{c}\text { Alterações da } \\
\text { deglutição }\end{array}$} & $\begin{array}{l}\text { Consistência do } \\
\text { alimento }\end{array}$ \\
\hline C1 & Ausência de alterações & $\begin{array}{l}\text { Todas as } \\
\text { consistências }\end{array}$ \\
\hline C2 & Ausência de alterações & $\begin{array}{l}\text { Todas as } \\
\text { consistências }\end{array}$ \\
\hline C3 & $\begin{array}{l}\text { Ausculta cervical positiva } \\
\text { Pigarro/tosse } \\
\text { Engasgo } \\
\text { Qualidade vocal } \\
\text { molhada }\end{array}$ & Líquidos* \\
\hline C4 & Ausência de alterações & $\begin{array}{l}\text { Todas as } \\
\text { consistências }\end{array}$ \\
\hline C5 & Ausência de alterações & $\begin{array}{l}\text { Todas as } \\
\text { consistências }\end{array}$ \\
\hline C6 & $\begin{array}{l}\text { Mastigação inadequada } \\
\text { Tempo de trânsito oral } \\
\text { aumentado }\end{array}$ & $\begin{array}{l}\text { Semissólido e } \\
\text { sólido }\end{array}$ \\
\hline
\end{tabular}

Nota: *Neste caso, por conta do ruim desempenho em avaliaçáo direta com líquido, não foi dada continuidade à avaliaçấo com outras consistências.

Após evento cirúrgico e estabilidade clínica, foi realizada nova avaliação fonoaudiológica. As avaliaçôes ocorreram um a dois dias após a cirurgia.

No momento da avaliação no período pós-operatório, cinco casos encontravam-se em adequado nível de consciência e um apresentou nível de consciência alterado. Em todos os casos, os pacientes encontravam-se em ventilação espontânea ao ar ambiente. Quanto à via de alimentação no pós-operatório imediato, dois casos estavam com dieta normal por via oral exclusiva, um estava com dieta pastosa (semissólida) por via oral exclusiva, outro estava em dieta semilíquida por via oral exclusiva, e dois encontravam-se em uso de sonda nasoenteral como via alternativa de alimentação exclusiva. A compreensão e a expressão no pós-operatório foram consideradas normais em quatro casos. Em um, considerou-se compreensão normal e expressão alterada e, em outro, foram observados déficits na comunicação nos aspectos da compreensão e expressão.

Com relação aos órgãos fonoarticulatórios, dois casos apresentaram musculatura facial sem alteraçôes, dois paresia facial à direita, um paresia facial à esquerda, e outro paresia facial bilateral. Quanto à força e mobilidade de estruturas orofaciais (lábios, língua e bochechas), quatro apresentaram todos os parâmetros alterados, um apresentou reduçáo de mobilidade e força de bochechas e outro, reduçáo de mobilidade de língua. Em avaliação funcional, cinco casos apresentaram frequência de deglutição de saliva espontânea adequada, com ausculta cervical limpa, e um caso apresentou ausência de 
deglutição de saliva espontânea e sob comando, com presença de roncos de transmissão em ausculta cervical.

A tosse sob comando foi considerada forte em dois casos e fraca em três, em um caso náo foi observada produção de tosse sob comando, porém a tosse induzida por manipulação laríngea foi considerada forte.

A qualidade vocal foi classificada como grau global 1 com discreta rugosidade para cinco casos e em um não foi possível realizar avaliação vocal, em razão da ausência de respostas sob comandos verbais.

Foi realizada avaliação direta de deglutição de alimentos em cinco casos e, em um caso, não foi possível prosseguir com avaliação por causa da alteração do nível de consciência e desempenho insatisfatório em avaliação indireta. Os resultados encontrados foram descritos na Tabela 2. Cinco casos obtiveram diagnóstico de disfagia leve e, em um caso, foi observada disfagia severa. A FOIS foi reaplicada no pós-cirúrgico e cinco casos foram classificados como nível 6 (via oral total com múltiplas consistências sem necessidade de preparo especial ou compensaçôes, porém com restriçôes alimentares) e um classificado como nível 1 (nada por via oral). A conduta fonoaudiológica pós-cirúrgica quanto à via de alimentação foi definida. A dieta oral exclusiva na consistência semissólida foi liberada em cinco casos; em um caso, foi contraindicada dieta oral.

$\mathrm{Na}$ Tabela 3, foram categorizadas as principais alteraçóes fonoaudiológicas encontradas em cada caso, comparando os dois momentos de avaliação. Nesta tabela, foram consideradas a Escala GRBASI, paralisia/paresia facial, triagem da comunicação compreensão e expressão), Escala FOIS e classificação da deglutição.

Em todos os casos do estudo, foi necessária terapia fonoaudiológica após o procedimento cirúrgico.

Tabela 2. Relações entre as alterações de deglutição e consistência do alimento no pós-operatório

\begin{tabular}{l|l|l}
\hline Caso & \multicolumn{1}{|c|}{$\begin{array}{c}\text { Alteraçães da } \\
\text { deglutição }\end{array}$} & $\begin{array}{l}\text { Consistência } \\
\text { do alimento }\end{array}$ \\
\hline C1 & $\begin{array}{l}\text { Mastigação inadequada } \\
\text { Tempo de trânsito oral } \\
\text { aumentado } \\
\text { Resíduo oral } \\
\text { Deglutições múltiplas }\end{array}$ & $\begin{array}{l}\text { Semissólido } \\
\text { e sólido }\end{array}$ \\
\hline C2 & $\begin{array}{l}\text { Mastigação inadequada } \\
\text { Tempo de trânsito oral } \\
\text { aumentado }\end{array}$ & $\begin{array}{l}\text { Semissólido } \\
\text { e sólido }\end{array}$ \\
\hline C3 & $\begin{array}{l}\text { Sem critérios para } \\
\text { avaliação direta da } \\
\text { deglutição }\end{array}$ & - - - \\
\hline C4 & $\begin{array}{l}\text { Mastigação inadequada } \\
\text { Tempo de trânsito oral } \\
\text { aumentado }\end{array}$ & $\begin{array}{l}\text { Semissólido } \\
\text { e sólido }\end{array}$ \\
\hline C5 & $\begin{array}{l}\text { Tempo de trânsito oral } \\
\text { aumentado }\end{array}$ & $\begin{array}{l}\text { Semissólido } \\
\text { e sólido }\end{array}$ \\
\hline \multirow{2}{*}{ C6 } & $\begin{array}{l}\text { Mastigação inadequada } \\
\text { Tempo de trânsito oral } \\
\text { aumentado } \\
\text { Resíduo oral } \\
\text { Deglutições múltiplas }\end{array}$ & $\begin{array}{l}\text { Semissólido } \\
\text { e sólido }\end{array}$ \\
\hline
\end{tabular}

Tabela 3. Características demográficas e socioeconômicas dos participantes do estudo

\begin{tabular}{|c|c|c|c|c|c|c|c|c|c|c|}
\hline & \multicolumn{2}{|c|}{ Voz } & \multicolumn{2}{|c|}{ Motricidade orofacial } & \multicolumn{2}{|c|}{ Comunicação } & \multicolumn{2}{|c|}{ Escala FOIS } & \multicolumn{2}{|c|}{ Classificação da deglutição } \\
\hline & Pré & Pós & Pré & Pós & Pré & Pós & Pré & Pós & Pré & Pós \\
\hline $\mathrm{Cl}$ & GOROBOAOSOIO & GORIBOAOSOIO & $\begin{array}{c}\text { Sem } \\
\text { alterações }\end{array}$ & $\begin{array}{c}\text { Paresia facial } \\
\text { Direita }\end{array}$ & $\begin{array}{c}\text { Sem } \\
\text { alterações }\end{array}$ & $\begin{array}{c}\text { Sem } \\
\text { alterações }\end{array}$ & 7 & 6 & $\begin{array}{c}\text { Deglutição } \\
\text { normal }\end{array}$ & Disfagia leve \\
\hline $\mathrm{C} 2$ & GOROBOAOSOIO & GORIBOAOSOIO & $\begin{array}{c}\text { Sem } \\
\text { alterações }\end{array}$ & $\begin{array}{c}\text { Paresia facial } \\
\text { Direita }\end{array}$ & $\begin{array}{c}\text { Sem } \\
\text { alterações }\end{array}$ & $\begin{array}{c}\text { Sem } \\
\text { alterações }\end{array}$ & 7 & 6 & $\begin{array}{c}\text { Deglutição } \\
\text { normal }\end{array}$ & Disfagia leve \\
\hline C3 & GOROBOAOSOIO & --- & Paresia facial & $\begin{array}{l}\text { Paresia } \\
\text { bilateral }\end{array}$ & $\begin{array}{l}\text { Alteração de } \\
\text { expressão }\end{array}$ & $\begin{array}{l}\text { Alteração de } \\
\text { compreensão } \\
\text { e expressão }\end{array}$ & 2 & 1 & $\begin{array}{c}\text { Disfagia } \\
\text { orofaríngea } \\
\text { moderada }\end{array}$ & Disfagia severa \\
\hline C4 & GORIBOAOSOIO & GORIBOAOSOIO & $\begin{array}{c}\text { Paresia facial } \\
\text { esquerda }\end{array}$ & $\begin{array}{c}\text { Paresia facial } \\
\text { esquerda }\end{array}$ & $\begin{array}{c}\text { Sem } \\
\text { alterações }\end{array}$ & $\begin{array}{c}\text { Sem } \\
\text { alterações }\end{array}$ & 7 & 6 & $\begin{array}{l}\text { Deglutição } \\
\text { funcional }\end{array}$ & Disfagia leve \\
\hline C5 & GOROBOAOSOIO & GOROBOAOSOIO & $\begin{array}{c}\text { Sem } \\
\text { alterações }\end{array}$ & $\begin{array}{c}\text { Sem } \\
\text { alterações }\end{array}$ & $\begin{array}{c}\text { Sem } \\
\text { alterações }\end{array}$ & $\begin{array}{c}\text { Alteração de } \\
\text { expressão }\end{array}$ & 7 & 6 & $\begin{array}{c}\text { Deglutição } \\
\text { normal }\end{array}$ & Disfagia leve \\
\hline C6 & GOROBOAOSOIO & GORIBOAOSOIO & $\begin{array}{c}\text { Sem } \\
\text { alterações }\end{array}$ & $\begin{array}{c}\text { Sem } \\
\text { alterações }\end{array}$ & $\begin{array}{c}\text { Sem } \\
\text { alterações }\end{array}$ & $\begin{array}{c}\text { Sem } \\
\text { alterações }\end{array}$ & 6 & 6 & $\begin{array}{c}\text { Disfagia } \\
\text { orofaríngea } \\
\text { leve }\end{array}$ & Disfagia leve \\
\hline
\end{tabular}

Legendas: G: grau global; R: rugosidade; B: soprosidade; A: astenia; S: tensăo; I: instabilidade. 


\section{DISCUSSÃO}

O meningioma é um dos tumores cerebrais primários adultos mais frequentes e representam 15\% dos tumores intracranianos e $30 \%$ de todos os tumores do sistema nervoso central provenientes das coberturas meníngeas da medula espinhal e do cérebro ${ }^{13}$. As alterações no período pós-operatório foram frequentes, principalmente quanto à deglutição fonoaudiológica, com involução da FOIS e necessidade de terapia fonoaudiológica em todos os casos.

Os dados deste estudo condizem com os achados da literatura ${ }^{4-5,14-16}$ que relatam um acometimento maior do meningioma em indivíduos do sexo feminino, na faixa etária de 40 a 70 anos. Quanto à escolaridade, a maioria tinha como formação o nível fundamental incompleto, dado que pode ser explicado pelo público-alvo geral do hospital onde foi realizado o estudo.

A literatura ${ }^{17}$ estabelece que $60 \%$ dos meningiomas intracranianos ocorrem adjacentes ao seio sagital superior, no córtex cerebral e ao longo da crista esfenoide. Desenvolvem-se com frequência também ao longo da base do crânio (meningioma do sulco olfativo, da sela turca, tentorial e petroclival). No presente estudo, a região cortical frontal foi a mais acometida, com ocorrência também na base do crânio, mais especificamente na asa do esfenoide, sela turca e fossa posterior. Portanto, são diversas as localizaçóes possíveis e, assim, os sintomas dependem da localização, assim como do crescimento, tamanho e do comprometimento do tecido cerebral ${ }^{14}$.

O tratamento definido pela maioria dos autores ${ }^{18-19}$ é o cirúrgico e a maior parte das séries publicadas relata uma taxa de ressecção total em torno de $70 \%$ dos $\operatorname{casos}^{18}$. O diagnóstico precoce é importante para que se atinjam os melhores resultados terapêuticos e para evitar que os tumores cheguem a áreas como os nervos cranianos e vasos ${ }^{20}$.

As deficiências neurológicas decorrentes das neoplasias meníngeas são causadas pela compressão de estruturas subjacentes ${ }^{4}$, deslocamento de estruturas cerebrais, aumento da pressão intracraniana, edema, manipulação cirúrgica ${ }^{14}$. As alteraçóes fonoaudiológicas são manifestaçóes comuns nos casos de tumoraçáo cerebral, com um espectro de déficits cognitivos, de comunicação, comportamentais e físicos, que complicam substancialmente o estado geral do paciente.

Após a manipulação cirúrgica, não foram observadas alteraçôes vocais significativas, apenas leve rugosidade, o que pode ser justificado pela intubaçấo orotraqueal de curta duração para procedimento cirúrgico ${ }^{21}$. Da mesma maneira, na literatura não é comum a associação de alteraçôes vocais ao meningioma. Um estudo ${ }^{22}$ mostra um raro caso de paralisia unilateral de pregas vocais em meningioma do forame jugular. As lesōes originadas nessa área têm um relacionamento íntimo com os nervos cranianos mais baixos, e, não comumente, podem surgir alguns sinais e sintomas resultantes da invasão dos nervos cranianos IX-XII, e incluem alteraçáo vocal ${ }^{22}$.

Já as alterações de motricidade orofacial, como a paresia facial, estiveram presentes na maioria dos casos no pós-operatório, assim como mostra a literatura ${ }^{8,23-24}$. Um estudo realizado ${ }^{23}$ relata que a manipulação cirúrgica nos casos de meningioma, muitas vezes, acarreta lesôes aos nervos cranianos envolvidos, levantando discussōes quanto à seleção da abordagem cirúrgica, principalmente no que diz respeito à gestão do nervo facial.

A comunicaçáo, considerando-se os aspectos compreensão e expressão, foi mais incisivamente prejudicada no caso do tumor da asa do esfenoide esquerda, com compressão das regióes frontal e temporal. Neste caso, foi realizada ressecção parcial do tumor, em virtude do envolvimento de vasos importantes. A literatura ${ }^{19}$ mostra que alteraçóes para realizar tarefas de conceituação e fluência lexical, memória de trabalho, flexibilidade mental, alterações neuropsicológicas podem ser associadas ao meningioma frontoparietal. Déficits cognitivos e linguísticos no pós-operatório em pacientes diagnosticados com meningioma em geral também foram citados, principalmente quanto à memória, atenção, expressão $^{3,14,25}$.

No que diz respeito à deglutiçáo, em todos os casos do estudo, foram obtidos déficits que levaram à involuçáo de consistência por via oral e redução da escala FOIS, com algum nível de disfagia orofaríngea. $\mathrm{O}$ caso do meningioma de asa do esfenoide também foi o mais devastador para a deglutiçáo. Um estudo realizado 9 mostra que, no pós-operatório imediato, houve evolução com broncopneumonia aspirativa secundária à paresia de nervos cranianos baixos. Os meningiomas de grande volume, situados na fossa posterior, cursam com deterioração grave e rapidamente progressiva ${ }^{9}$, envolvendo importantes estruturas vasculares e neurais ${ }^{22}$.

A avaliação fonoaudiológica antes e após o procedimento cirúrgico é essencial para identificação de alteraçóes funcionais, permitindo que sejam conduzidas condutas necessárias à reabilitação, assim como definido em outros estudos ${ }^{22-23}$.

\section{CONCLUSÃO}

Conclui-se que o meningioma pode acarretar diferentes alteraçóes fonoaudiológicas, principalmente quanto à deglutição, sendo mais frequentes no momento pós-cirúrgico em razão da manipulação dos vasos e nervos, e mais preocupantes em regiôes cranianas posteriores. A presença do fonoaudiólogo na equipe hospitalar é 
essencial, uma vez que este tem papel fundamental na adequação da via de alimentaçáo, identificando broncoaspiraçóes, minimizando complicaçóes, e também otimizando a comunicaçáo do paciente.

\section{CONTRIBUIÇÕES}

Caroline Sousa Costa contribuiu para a concepçáo do trabalho, interpretação dos dados da pesquisa, redação e revisão crítica, aprovação final da versão para submissão do artigo. Renata D'Arc Scarpel contribuiu para a concepção do trabalho, interpretação dos dados da pesquisa, revisão crítica e aprovação final da versão para submissão. Ana Terra Brito de Jesus contribuiu para a concepçáo do trabalho, interpretação dos dados da pesquisa, revisão crítica e aprovação final da versão para publicação.

\section{DECLARAÇÃO DE CONFLITOS DE INTERESSE}

Nada a declarar.

\section{REFERÊNCIAS}

1. Gasparini B. Mortalidade por tumores do Sistema Nervoso Central no município do Rio de Janeiro, 19802007. [Dissertação]. Rio de Janeiro: Escola Nacional de Saúde Pública Sergio Arouca; 2010. 77 p.

2. Pieri JN, Giriko CH, Abranches MHS, Borges HC, Chamlian TR. Assesment of functional independence and quality of life after surgery for brain tumor. Rev Neurocienc. 2011; 9(3):477-483.

3. Nayar VV, DeMonte F, Yoshor D, Blacklock JB, Sawaya R. Surgical approaches to meningiomas of the lateral ventricles. Clinical Clin Neurol Neurosurg. 2010; 112(5): 400-405.

4. Kirshblum, S. O’Dell MW, Ho C, Barr K. Rehabilitation of persons with central nervous system tumors. Cancer. 2001; 92 (4 Suppl): 1029-38.

5. Silva DOA, Costa LF, Kitamura MP, Silva JC, Azevedo Filho HRC. Meningiomas do forame magno: revisão de literatura. Arq. Bras Neurocir. 2009; 28(2):74-80.

6. Commins D, Atkinson RD, Burnett ME. Review of meningioma histopathology. Neurosurg Focus. 2007;23(4).

7. Landeiro, JA, Ribeiro CH, Lapenta MA, Flores MS, Lopes CAR, Marins J. Meningiomas do seio cavernoso: Correlação entre a extensão de ressecção cirúrgica e lesóes neurovasculares em 16 pacientes. Arq. Neuropsiaquiatr. 2001;59(3): 746-753.

8. Nakamura M, Roser F, Bundschuh O, Vorkapic P, Samii M. Intraventricular meningiomas: a review of 16 cases with reference to the literature. Surg Neurol. 2003; (59):491-504.
9. Tella JR, OI Paiva Neto MA, Aguiar PH, Herculano MA. Meningiomas anteriores e antero-laterais do forame magno. Arq Neuropsiquiatr.2006; 64(2):437-440.

10. Padovani AR, Moraes DP, Mangili LD, Andrade CRF. Protocolo fonoaudiológico de avaliação do risco para disfagia (PARD). Rev. Soc. Bras. Fonoaudiol.2007; 12(3):199-205.

11. Piccirilo JF, Painter C, Fuller D, Haiduk A, Fredrickson JM. Assessment of two objective voice function índices. Ann. Otol. Rhinol. Laryngol. 1998; 107(5):386-400.

12. Crary MA, Mann GD, Groher ME. Initial psychometric assessment of a functional oral intake scale for dysphagia in stroke patients. Arch Phys Med Rehab. 2005; 86(8):1516-20.

13. Ohla V, Scheiwe C. Meningiomatosis restricted to the left cerebral hemisphere with acute clinical deterioration: case presentation ans discussion of treatmente options. Surg Neurol Int. 2015; 6:64.

14. Miotto, EC. Marie SKN, Teixeira MJ, Aguiar PHP. Déficits cognitivos em pacientes com meningiomas. J. Bras. Neurocirurg. 2003; 14(3):87-91.

15. Viegas SFJ. Tumor cerebral: Incidência, diagnóstico e tratamento. [Dissertação]. Algarve: Faculdade de Ciências e tecnologia; 2010. 35p.

16. Wiemels J. Wrensh M, Claus EB. Epidemiology and etiology of meninfioma. J Neurooncol. 2010; 99(3):307-314.

17. Afumu CN, Ramos JFRV, Rodríguez OL, Rodríguez IA, Corrales AMB. Los meningiomas intracraniales recidivantes postquirúrgicos. Rev. Ciencias Medicas. 2014; 18 (2): 231-243.

18. Bunyataravej K, Siwanuwatn R, Chantra $\mathrm{K}$, Khaoroptham S. Duration of symptoms in brain tumors: Influencing factors and its value in predicting malignant tumors. J Med Assoc Thai. 2010; 93(8): 903-910.

19. Hurtado-González CA, De la Cruz O, Calvo-Ortiz V, Olayo J, Triviño O, Brown-Castrillón P, Semine C. Neuropysichological abnormalities in a patient diagnosed with frontoparietal meningioma. Biomedical research. 2017; 28(2):701-704.

20. Sanai N, McDermott MW. A modified far-lateral approach for large or giant meningiomas of the posterior fossa. J Neurosurg, 2010; 112(5):907-12.

21. Campos NF, Bougo GC, Gama ACC, Vicente LCC. Efeitos da intubação orotraqueal na voz e deglutição de adultos e idosos. Distúrb Comum. 2016; 28(4): 597-60.

22. Senior A, Douglas JA, Thompson S. Unilateral vocal cord palsy and dysphagia: an unusua lpresentation of cerebellopontine angle meningioma. J Surg Case Rep. 2015;(10):1-3.

23. Gilbert ME, Shelton C, McDonald A, Salzman KL, Harnsberger HR, Sharma PK, et al. Meningioma 
of the jugular foramen: Glomus jugulare mimic ans surgical challenge. Laryngoscope. 2014; 114(1):25-32.

24. Bakar, B. Jugular Foramen Meningiomas: Review of the major surgical series. Neurol Med Chir. 2010; 50(2):89-97.

25. Meskal I, Gehring K, Rutten GJ, Sitskoorn MM Cognitive functioning in meningioma patients: a systematic review. J Neurooncol.2016; 128(2):195-205.

Recebido em 29/5/2018

Aprovado em 17/10/2018 


\title{
ANEXO 1. PROTOCOLO DE AVALIAÇÃO CLÍNICA FONOAUDIOLÓGICA À BEIRA LEITO
}

\author{
HOSPITAL GERAL ROBERTO SANTOS \\ SERVIÇO DE FONOAUDIOLOGIA
}

PROTOCOLO DE AVALIAÇÃO CLÍNICA FONOAUDIOLÓGICA À BEIRA LEITO

\begin{tabular}{|c|c|c|}
\hline Nome: & & \multirow{2}{*}{ Sexo: ( ) F( ) M } \\
\hline Unidade/leito: & \multirow[t]{2}{*}{ Unidade/leito: __ Registro: } & \\
\hline \multicolumn{2}{|l|}{ Data da avaliação: } & \\
\hline & & \\
\hline
\end{tabular}

\section{AVALIAÇÃO INDIRETA}

Estado de vigilância ( ) Sim ( ) Não (o paciente deve estar alerta por pelo menos 15 minutos)

Nível de consciência ( ) Alerta ( ) Alterado

Respiraçáo ( ) Espontânea $\mathrm{O}_{2} \_$( ) VMNI

Necessidade de aspiração ( ) não ( ) sim

Via de alimentaçáo ( ) VO consistência

Volume aspirado $\operatorname{Cuff}(+)(-)$

( ) Dieta zero

Comunicaçáo ( ) Compreensão （ ) Expressão [N: normal ou A: alterada]

\subsection{Avaliaçáo de OFA'S:}

Musculatura facial ( ) Normal ( ) Paralisia/Paresia Facial D/E Outros

Oroscopia ( ) Dentição permanente ( ) Prótese dentária ( ) Ausência de dentes parcial/total ( ) Higiene insatisfatória

Sialorreia ( ) ( ) Acúmulo de saliva em cavidade oral （ ) Xerostomia （ ) Lesôes em mucosa

Aspecto das estruturas (N: normal) ou (R: reduzido)

Lábios: Força ( ) Mobilidade ( ) Selamento labial em repouso ( )

Língua: Mobilidade ( ) Força ( ) Obs.:

Bochechas: Mobilidade: ( ) Força ( ) Obs.:

Palato mole: Mobilidade ( ) Obs.:

Excursão laríngea (mediçáo 2 dedos): ( ) Obs.:

\section{AVALIAÇÃO FUNCIONAL}

2.1 Deglutiçáo salivar: ( ) Adequada ( ) Alterada \{( ) diminuição da frequência ( ) ausência\}

2.2 Ausculta cervical: ( ) Limpa ( ) Presença de roncos de transmissão ( ) Sem possibilidade de avaliação - motivo

\subsection{Tosse e/ou pigarreio ( ) Sim [Espontânea/sob comando] Espontânea (+)(-) Comando (+)(-) ( ) Não}

* (+) Forte / (-) Fraca

2.4 Qualidade vocal: ( ) Sem alteraçôes ( ) Voz molhada ( ) Alterada *Escala G_ R_ B_ A_ S_ I_

* G- grau global; R- rugosidade; B- soprosidade; A- astenia; S- tensão; I- instabilidade/ Grau: 0- normal ou ausente;

1- discreto; 2- moderado; 3- severo

\section{SOMENTE PARA PACIENTE TRAQUEOSTOMIZADO}

Tipo de traqueostomia plástica/silicone ( ) metálica ( )

Tolera cuff desinsuflado ( ) Sim ( ) Não

Oclusão de TQT [Digital ( ) Sim ( ) Não // Embolo da seringa ( ) Sim ( ) Não //

Válvula de Fala ( ) Sim ( ) Não

Blue Dye Test ( + ) ( - ) ( ) Não se aplica 


\subsection{Impossibilidades para o teste de deglutiçáo com dieta}

( ) Nível de alerta ( ) Nível de consciência ( ) Mínimo de 24 horas após extubação (para IOT por período maior do que 48 horas)

( ) Recusa Alimentar ( ) Instabilidade clínica ( ) Dieta zero ( ) Desempenho não satisfatório em avaliação indireta ( ) Outros

3.2 Avaliaçáo direta da deglutição (Material: Suco, espessante, pão/ou fruta mole, biscoito, colher rasa, copo 50ml, seringa de $20 \mathrm{ml}$ )

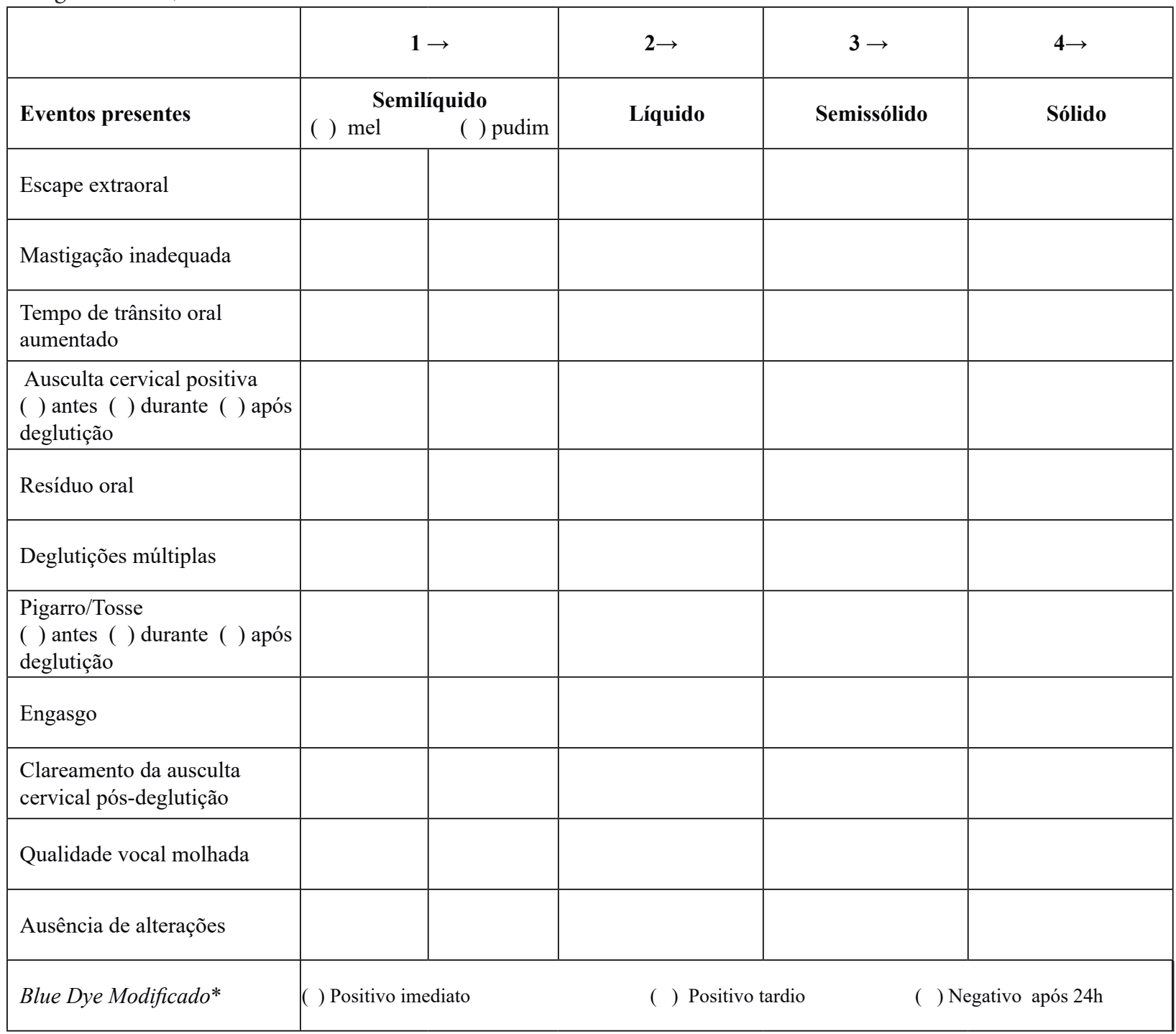

*Positivo: saída de secreção corada via traqueostomia

Negativo: sem saída de secreção corada via traqueostomia

\section{CONCLUSÃO}

Diagnóstico fonoaudiológico:

( ) Deglutiçáo normal ( )Deglutiçáo funcional ( ) Disfagia leve ( ) Disfagia moderada ( ) Disfagia grave ( ) Disfagia severa

Escala Funcional de Ingestáo por via oral (FOIS):

( ) Nível 1: Nada por via oral

( ) Nível 2: Dependente de via alternativa e mínima via oral de algum alimento ou líquido

( ) Nível 3: Dependente de via alternativa com consistente via oral de alimento ou líquido

( ) Nível 4: Via oral total de uma única consistência

( ) Nível 5: Via oral total com múltiplas consistências, porém com necessidade de preparo especial ou compensaçôes 
( ) Nível 6: Via oral total com múltiplas consistências, porém sem necessidade de preparo especial ou compensações, porém com restriçôes alimentares

( ) Nível 7: Via oral total sem restriçóes

\section{CONDUTA}

( ) Contraindicação de via oral.

( ) Fonoterapia indireta.

( ) Treino de via oral

( ) Dieta via oral, consistências Liberadas:

( ) Líquida ( ) Semilíquida néctar/mel/pudim ( ) Semissólido ( ) Sólido ( ) Todas as consistência sem restriçóes

Modo de oferta: ( ) Colher （）Copo （）Canudo （）Sem restrições （）Assistida/Supervisionada ( ) Independente.

Observação:

Fonoaudiólogo Responsável:

Autoria: Jesus, A.T.B. Costa, C.S., Santos, C.B., Barbosa, C.R., Pinheiro, E.N.S, Gomes,J.K.S. Colaboração:

Torres,A.C, Sousa, C.S 